\title{
Physical Symmetries in a Theory of Several Scalar Real Fields
}

\author{
JAN T. ŁOPUSZAŃSKI \\ Institute of Theoretical Physics, University of Wroclaw, Wroclaw
}

Received March 27, in revised version June 2, 1969

\begin{abstract}
Let us consider a theory of $n$ scalar, real, local, Poincaré covariant quantum fields forming an irreducible set and giving rise to one particle states belonging to the same mass different from zero. The vacuum is unique. It is shown under fairly weak assumptions that every Poincare and TCP invariant symmetry of the theory, implemented unitarily, which mapps localized elements of the field algebra into operators almost local with respect to the former (such a symmetry we call a physical one) can be defined uniquely in terms of the incoming or outgoing fields and an $n$-dimensional (real) orthogonal matrix. The symmetry commutes with the scattering matrix. Incidentally we show also that the symmetry groups are compact. A special case of these symmetries are the internal symmetries and symmetries induced by locally conserved currents local with respect to the basic fields and transforming under the same representation of the Poincare group. We may make linear combinations out the original fields resulting in complex fields and its complex conjugate in a suitable way. The inspection of the representations of the groups $S O(n)$ and their subgroups sheds some light on the s.c. generalized Carruthers Theorem concerning the self- and pair-conjugate multiplets.
\end{abstract}

1. It is of some interest to explore the problem of physical symmetries starting from a theory of $n$ scalar, real fields subjected to some fairly weak restrictions (see below). By a physical symmetry we mean not only internal symmetries (preserving strict locality) but a larger class of symmetries mapping localized elements of the field algebra into operators almost local with respect to the former [1]. We believe that every symmetry induced by a locally conserved current belongs to his class and there are strong indications that it is so (see $[2,3]$ ).

We succeeded in giving the recipe how to find the most general of such a symmetry, provided it is unitarily implemented in the Hilbert space. It commutes with the scattering matrix. Incidentally we showed also that the symmetry groups are compact.

Linear rearrangement of the original fields, suitable for physical problems, yields then a possibility of a classification of the relevant symmetries occuring in the theory of $n$ scalar (not necessarily real) fields. The present results encompass the results obtained earlier by the author [2]. It is worthwhile to notice that some light is shed 
on the problem of self- and pair-conjugate multiplets raised by Carruthers (s. c. generalized Carruthers theorem [4]).

2. To start with we list the relevant assumptions

i) The Poincare group is unitarily implemented in the Hilbert space $\mathscr{H}$. We denote the representation of $(\Lambda, a)$ by $U(\Lambda, a)$. The spectrum of $P^{2}$, where

$$
T(a)=U(\mathbf{1}, a)=\exp \{i P a\}
$$

consists of two discrete points $\mu^{2}=0$ (corresponding to the single vacuum state $\Omega$ ) and $\mu^{2}=m^{2} \neq 0$ (corresponding to one particle states) as well as out of a continuous part, say $\left(4 \mathrm{~m}^{2}, \infty\right)$ (scattering states).

ii) We are concerned with $n$ scalar, real quantum fields $\psi_{j}(x)$ $j=1, \ldots n$. All of them transform under the same Poincaré representation, viz.

$$
U(\Lambda, a) \psi_{j}(x) U(\Lambda, a)^{+}=\psi_{j}(\Lambda x+a), \quad j=1, \ldots n
$$

are local and local with respect to each other ${ }^{1}$. We assume for simplicity reasons that

where

$$
\left(\Omega, \psi_{j}(f) \Omega\right)=0, \quad j=1, \ldots n
$$

$$
\psi_{j}(f)=\int \psi_{j}(x) f(x) d x, \quad f(x) \in \mathscr{S}_{4} .
$$

iii) To exclude trivial cases and to get proper asymptotic conditions [5] we assume that

$$
\sum_{j=1}^{n} a_{j} E_{1} \psi_{j}(f) \Omega=0 \quad \text { iff } \quad a_{j}=0 .
$$

Here $E_{1}$ is the projection operator on the Hilbert subspace $\mathscr{H}$, labelled by $p^{2}=m^{2}, p_{0}>0$ (spanned by the one particle states induced by the fields $\psi_{j}(x)$ ).

iv) Then the free asymptotic fields [6] exist and we assume them to be irreducible. This implies that the original set of $n$ fields is also irreducible. As a matter of fact it would be sufficient to assume only that the incoming fields form an irreducible set (due to the existence of a common TCP operator).

We may form linear combinations out of these fields. They will be again local, local with respect to each other, real, will transform under the same representation of the Poincare group as $\psi_{j}$ and build an irreducible set. Let us call them $\varphi_{j}(x) j=1, \ldots, n$. We demand in addition that ${ }^{2}$

$$
\left(\Omega, \varphi_{j}(x) E_{1}, \varphi_{k}(y) \Omega\right)=i \delta_{j k} \Delta^{(+)}\left(x-y ; m^{2} ; j, k=1, \ldots n\right.
$$

\footnotetext{
${ }^{1}$ We believe that weak locality would be sufficient for our aims.

2 The case $\left(\Omega, \varphi_{j}(x) E, \varphi_{k}(y) \Omega\right)=0$ is excluded by the assumption (3).
} 
These fields have asymptotic free fields

$$
\varphi_{j, \text { in }}(x) \equiv \varphi_{j, \text { ex }}(x) \quad(\text { in virtue of (3)) }
$$

belonging all to the same mass $m^{2} \neq 0$.

We have the

Statement. Let us assume that there exists such a unitary transformation $V$ for which

$$
(\Omega, V \Omega) \neq 0
$$

and the fields

$$
V \varphi_{j}(x) V^{+} \equiv A_{j}(x), \quad j=1, \ldots n
$$

transform under the same representation of the Poincaré group and under the same TCP operator ${ }^{3}$ as $\varphi_{j}(x)$, viz.

$$
\begin{gathered}
U(\Lambda, a) A_{j}(x) U(\Lambda, a)^{+}=A_{j}(\Lambda x+a), \quad j=1, \ldots n \\
\Theta A_{j}(x) \Theta^{-1}=A_{j}(-x)
\end{gathered}
$$

and are almost local with respect to $\varphi_{k}(x), k=1, \ldots n$. Then we have

$$
V \varphi_{j, \mathrm{ex}}(x) V^{+}=\sum_{k=1}^{n} \alpha_{j k} \varphi_{k, \mathrm{ex}}(x)
$$

where $\alpha_{j k}$ forms an $n$-dimensional (real) orthogonal matrix and

$$
[V, S]=0
$$

where $S$ is the scattering matrix. $V$ is uniquely defined by (8).

Proof. From the fact that $\varphi_{j}$ and $A_{k}$ transform under the same representation of the Poincare group and from the irreducibility of the fields $\varphi_{j}$ as well as from (5) follows

$$
[U(\Lambda, a), V]=0
$$

and $V \Omega=e^{i b} \Omega, b$ a real number.

To get rid of the factor $e^{i b}$ we redefine $V$ in such a way that

$$
V \Omega=\Omega .
$$

Let $V$ act on the one particle states

$$
E_{1} \varphi_{j}(f) \Omega \equiv \Phi_{j}(f) .
$$

${ }^{3}$ The author is grateful to Dr. M. Rinke who pointed out that a common TCP operator is sufficient to exclude cases like that of two real fields $\varphi_{\mathrm{ex}}(x)=\varphi_{\mathrm{ex}}^{(+)}(x)+\varphi_{\mathrm{ex}}^{(-)}(x)$ and $A_{\mathrm{ex}}(x)=e^{i \lambda} \varphi_{\mathrm{ex}}^{(+)}(x)+e^{-i \lambda} \varphi_{\mathrm{ex}}^{(-)}(x)$ which are qusilocal for every $\lambda$. 
In virtue of (10), irreducibility of the fields $\varphi_{j}(f)$ on the one particle subspace and the unitarity of $V$ we get

and

$$
\begin{gathered}
V \Phi_{j}(f)=\sum_{k=1}^{n} \alpha_{j k} \Phi_{k}(f), \\
V^{+} \Phi_{j}(f)=\sum_{k=1}^{n} \overline{\alpha_{k j}} \Phi_{k}(f), \\
\sum_{k=1}^{n} \overline{\alpha_{j k}} \alpha_{l k}=\delta_{j l} .
\end{gathered}
$$

Now the generalized result of Borchers [7] implies

$$
\left[\varphi_{j, \mathrm{ex}}(x), A_{k, \mathrm{ex}}(y)\right]=i \lambda_{j k} \Delta\left(x-y ; m^{2}\right)
$$

Making use of (12) and (13a) we see that

$$
\lambda_{j k}=\alpha_{j k} .
$$

The irreducibility of the fields $\varphi_{j, \text { ex }}$ as well as (11) lead us to the conclusion that

$$
A_{k, \mathrm{ex}}(x) \equiv V \varphi_{k, \mathrm{ex}}(x) V^{+}=\sum_{j=1}^{n} \alpha_{k j} \varphi_{j, \mathrm{ex}}(x)
$$

Since $\varphi_{k, \mathrm{ex}}$ are real fields

$$
\alpha_{k j}=\overline{\alpha_{k j}} .
$$

The matrix $\alpha_{k j}$ is then an $n$-dimensional (real) orthogonal matrix. Notice that $V$ is uniquely defined by (8) due to the irreducibility of the fields $\varphi_{k, \text { ex }}$ as well as to (11). Since (8) holds for the incoming as well as for the outgoing fields

$$
[V, S]=0 .
$$

This accomplishes the proof.

Thus the most general unitary transformation satisfying the hypotheses of the Statement is given by (8), (13) and (9).

From the Statement follows immediately the

Corollary. If the set of the unitary matrices $V(g)$ satisfying the hypotheses of the Statement forms a representation of a continuous group $G(g \in G)$ the group is compact.

Proof. The matrices $\alpha_{j k}(g)$ form an $n$-dimensional unitary representation of the group $G$.

The only admissible groups of physical symmetries are compact. 
3. The internal symmetries or physical symmetries whose generators are induced by a (pseudo) vectors [2] fall into the class of unitary transformations obeying the requirements of the Statement. For generators

$$
Q^{\mu}=\int T^{0 \mu}(x) d^{3} x ; \quad \mu=1,2,3,0
$$

induced by a (pseudo) tensors $T^{\mu v}(x)$ [3], local with respect to the original fields (it follows then that $T^{\mu \nu}(x)$ are local with respect to themselves), locally conserved, viz.

$$
\frac{\partial}{\partial x^{\lambda}} T^{\lambda \mu}(x)=0
$$

transforming according to

$$
U(\Lambda, a) T^{\lambda \mu}(x) U(\Lambda, a)^{+}=\Lambda^{\lambda} \sigma \Lambda^{\mu} \varrho T^{\sigma \varrho}(\Lambda x+a)
$$

the conditions imposed in the Statement (see conditions (7)) lead to $\alpha_{j k}=0$.

The same holds true for any generator set of tensorial character in the Minkowski space of rank $n \geqq 1$.

4. Special cases of the general procedure presented above are the cases considered in [2].

For $n=1$ we deal only with one scalar real field $\varphi$ and

$$
V \varphi_{\mathrm{ex}} V^{+}= \pm \varphi_{\mathrm{ex}}
$$

since according to (13) $|\alpha|^{2}=1$ and $\alpha=\bar{\alpha}$. There is no continuous group of symmetry transformations different from 1 .

In case $n=2$ we have the 2-dimensional real representation of the one parameter group $S O$ (2). We may introduce the field

$$
C=\frac{1}{\sqrt{2}}\left(\varphi_{1}+i \varphi_{2}\right)
$$

and its complex conjugate. Then

$$
V(\varphi) C_{\mathrm{ex}}(x) V(\varphi)^{+}=e^{i \varphi} C_{\mathrm{ex}}(x), \quad \varphi \text { real number }
$$

and $V(\varphi)$ is the gauge transformation. In case $n=2$ for any $V$ we have either (19) or

$$
V C_{\mathrm{ex}}(x) V^{+}=e^{i \beta} C_{\mathrm{ex}}^{+}(x), \quad \beta \text { real number }
$$

(discrete symmetry). A transformation like

$$
V C_{\mathrm{ex}}(x) V^{+}=f\left(x, C_{\mathrm{ex}}, C_{\mathrm{ex}}^{+}\right)
$$

is automatically ruled out. 
In case $n=3$ we may introduce the fields

$$
\begin{aligned}
& C_{1}=\frac{1}{\sqrt{2}}\left(\varphi_{1}+i \varphi_{2}\right)=C_{3}^{+}, \\
& C_{2}=\varphi_{3},
\end{aligned}
$$

and consider the 3-dimensional complex irreducible representation of $S O$ (3). In this way we get a triplet encompassing particles and its antiparticles as well, like in case of $\pi^{+}, \pi^{\circ}, \pi^{-}$. There is no 2-dimensional group transforming $C_{1}$ and $C_{3}$ among themselves, viz.

$$
V C_{1, \mathrm{ex}}(x) V^{+}=u C_{1, \mathrm{ex}}+v C_{3, \mathrm{ex}}, \quad u v \neq 0
$$

leaving $C_{2}$ unchanged. Should $C_{2}$ be left unchanged $u v$ has then to vanish and the case reduces to the previous one, (19) and (20).

In case $n=4$ we may introduce the fields

$$
\begin{aligned}
& C_{1}=\frac{1}{\sqrt{2}}\left(\varphi_{1}+i \varphi_{2}\right)=C_{3}^{+}, \\
& C_{2}=\frac{1}{\sqrt{2}}\left(\varphi_{3}+i \varphi_{4}\right)=C_{4}^{+} .
\end{aligned}
$$

Then in addition to the 6-parameter 4-dimensional complex irreducible representation of $S O(4)$ we may also consider the 3-parameter 2dimensional irreducible representation of $S U$ (2) and its complex conjugate. The latter ones will transform $C_{1}, C_{2}$, and $C_{3}, C_{4}$ resp. among themselves, viz.

$$
\begin{aligned}
& C_{1}^{\prime}=\alpha C_{1}+\beta C_{2} \\
& C_{2}^{\prime}=\gamma C_{1}+\delta C_{2}
\end{aligned} \quad \alpha, \beta, \gamma, \delta \quad \text { complex }
$$

Thus we get two doublets of particles no one of which will involve its antiparticles, like in case of $K^{+}$and $K^{o}$. However, there is no group transforming the fields $C_{1}$ and $C_{3}=C_{1}^{+}$among themselves. Other groups are trivial extensions of the cases considered earlier.

In case of $S O$ (3) we have to do with a self-conjugate and in case of $S U(2) \oplus S U(2)^{*}$ with a pair-conjugate multiplet. Of course, in the first case there exists a (3-dimensional) unitary matrix transforming the complex representation into a real one. In the latter such a 2-dimensional matrix does not exist.

These conclusions extended to the case of an $n$-dimensional representation of $S O(n), n$ - an arbitrary natural number, and its subgroups express the generalized Carruthers theorem [4] which can be formulated as follows: in case of a self-conjugate multiplet there exists a unitary 
matrix making the matrix representation of the group to a real one, in case of a pair-conjugate multiplet this can not be done (originally it was shown that self-conjugate isospin multiplets of bosons with half-integral isospin can not exist).

Acknowledgement. I am grateful to Mr. A. Z. Jadczyk for discussions.

\section{References}

1. Haag, R., and D. Kastler: J. Math. Phys. 5, 848 (1964).

2. Łopuszański: Commun. Math. Phys., in press, Reports on Mathematical Physics, in press.

3. Orzalesi, C. A., J. Sucher, and C. H. Woo: Phys. Rev. Letters 21, 1550 (1968).

4. Carruthers, P.: Phys. Rev. Letters 18, 353 (1967). - Jin, Y. S.: Phys. Letters 24 B, 411 (1967). — Fleming, G. N., and E. Kazes: Phys. Rev. Letters 18, 764 (1967). Lee, H.: Phys. Rev. Letters 18, 1098 (1967). - Kantor, P. B.: Phys. Rev. Letters 19, 394 (1967). - Fabri, E., and L. E. Picasso: Nuovo Cimento (in press).

5. Haag, R.: Phys. Rev. 112, 669 (1958). — Hepp, K.: Helv. Phys. Acta 37, 639 (1964). Hepp, K.: Commun. Math. Phys. 1, 111 (1965). - Araki, H., and R. Haag: Commun. Math. Phys. 4, 77 (1967).

6. For a definition of a free field see Streater, R. S., and A. S. Wightman: PCT, spin and statistics and all that. New York-Amsterdam: Benjamin 1964.

7. The original version due to Borchers, H. J.: Nuovo Cimento (X) 15, 784 (1960) was hereafter generalized by Araki, H.; see Hepp, K.: Lecture Notes of the Brandeis Summer School, Waltham, 1965, as well as Araki, H., R. Haag, and B. Schroer: Nuovo Cimento (X) 19, 90 (1961).

Jan T. Łopuszański

Institute of Theoretical Physics

University of Wroclaw

ul. Cybulskiego 36

Wroclaw, Poland 